Boletín de la Sociedad Geológica Mexicana

VOLUMEN 65, NÚM. 1, 2013, P. 1-8

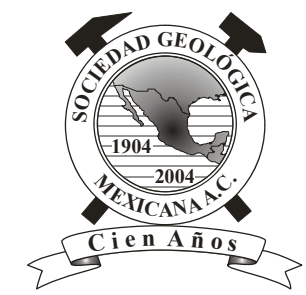

\title{
El método de Polarización Inducida aplicado en la contaminación de suelos por hidrocarburos
}

\author{
Andrés Tejero Andrade ${ }^{1, *}$, Aide Esmeralda López González ${ }^{1}$ \\ ${ }^{1}$ Universidad Nacional Autónoma de México, Facultad de Ingeniería, Av. Ciudad Universitaria No. 3000, Col. Universidad, \\ Delegación Coyoacán, C.P. 04510. \\ *atatejero16@gmail.com.mx
}

\section{Resumen}

Las pruebas obtenidas por algunos autores dedicados a los métodos eléctricos han mostrado que al realizar un levantamiento convencional de resistividad, no ha sido posible diferenciar zonas que presentan comportamientos electroquímicos semejantes, tanto de origen orgánico como inorgánico. Es por ello que en años recientes, el método de Polarización Inducida (PI) se ha aplicado a problemas de contaminación en suelos con resultados alentadores. Esta técnica ha sido puesta a prueba, trabajando en el dominio del tiempo, mediante un perfil de tomografía eléctrica con datos de resistividad y cargabilidad, el cual fue realizado en un sitio contaminado por hidrocarburos en una zona ubicada al norte de la Ciudad de México, en donde la estratigrafía y la distribución del contaminante eran complejas.

Los hidrocarburos se encontraban en fase libre y fase disuelta, en proceso de remediación, mismo que proporcionó las condiciones para la toma de muestras de suelo y agua que fueron enviadas a un laboratorio para su análisis químico, con lo que se obtuvieron concentraciones de benceno en cuatro pozos cercanos a la línea del perfil eléctrico.

Los resultados obtenidos muestran que se puede delimitar las zonas de mayor concentración de contaminantes, mediante la correlación de la información eléctrica, y capacitiva, y de manera cualitativa, con la información de los parámetros Cole-Cole, se demuestra el potencial del método de polarización inducida en problemas de contaminación

Palabras clave: tomografía de resistividad eléctrica, polarización, cargabilidad, hidrocarburos, contaminación.

\begin{abstract}
The evidence obtained by some authors devoted to electrical methods has shown that when a conventional resistivity survey is performed, it is not possible to differentiate areas with similar electrochemical behavior of both the organic and inorganic origin. Therefore, in recent years the method of Induced Polarization (IP) has been applied to soil contamination problems, showing encouraging results. This technique was tested, working in the time domain through an electrical tomography profile of resistivity and chargeability data, which was conducted at a site contaminated by hydrocarbons located in the north of Mexico City, where stratigraphy and distribution of the contaminant were complex.

The hydrocarbons were found in a free and dissolved phase, which provided the conditions for taking soil and water samples. These samples were sent to a laboratory for chemical analysis, which depicted benzene concentration in four wells.

The obtained results show that it is possible to define areas of large concentration of pollutants, by correlating electrical information, capacitive and qualitatively, with the information from the Cole-Cole parameters, demonstrating the potential of induced polarization method in pollution problems.
\end{abstract}

Keywords: electrical resistivity tomography, polarization, chargeability, hydrocarbons, pollution.. 


\section{Introducción}

A lo largo del desarrollo de la teoría y técnica de la prospección eléctrica, se ha buscado la manera de obtener las respuestas del subsuelo que aporten información suficiente para delimitar cuerpos de interés industrial como son metales, carbón, materiales de construcción, etcétera. En años recientes la contaminación de los suelos ha motivado la investigación de técnicas que permitan delinear zonas contaminadas, donde la Polarización Inducida (PI) ofrece resultados alentadores.

Los métodos para obtener respuestas de PI han sido modificados a través del tiempo y han dependido de la capacidad tecnológica, así como de variables que poco a poco se han considerado, como la granulometría y propiedades electroquímicas, que formaron parte de algunos modelos matemáticos que pretendieron describir mejor el comportamiento eléctrico, como los propuestos por Seigel (1959), Wong (1979) y Pelton et al. (1978).

De acuerdo al diccionario de la Sociedad de Geofísicos de Exploración (SEG, por sus siglas en inglés) (Sheriff, 2002), se define a la polarización inducida como el momento dipolar de la corriente por unidad de volumen. Se le atribuye ese nombre debido a que el comportamiento físico en la tierra funciona como un sistema capacitivo, que se "carga" o "polariza" al suministrar energía eléctrica al medio. Wong (1979) por su parte, lo define como la dispersión en bajas frecuencias, de la conductividad o resistividad entre los materiales de la tierra y el agua que se encuentran en contacto.

Con el paso de corriente eléctrica a través de la roca se crea un campo eléctrico $E$ que a su vez, puede producir uno o varios tipos de polarización dependiendo de su génesis: por acumulación de cargas (interfacial), por movimiento de cargas (electrónica), por desplazamiento de iones en la red cristalina (iónica) o bien, por la orientación de las moléculas (bipolar).

En términos generales existen dos tipos de polarización: la polarización electrónica y la polarización de membrana. La primera, supone que los poros del medio que están siendo ocupados por la solución, se encuentran bloqueados por partículas metálicas y la conducción de la corriente que era iónica, se convierte en conducción eléctrica en el metal. Así, la superficie existente entre el metal y el fluido experimenta una polarización. La polarización de membrana de acuerdo con Sogade et al. (2006), es causada por la impedancia de interfase entre dos medios que resulta cuando, en ciertas regiones de conducción normal en una trayectoria de poro, una capa de difusión es lo suficientemente gruesa para bloquear dicho poro, de manera que hay una transferencia de iones de cierto tamaño y polaridad, reduciendo la movilidad de las cargas causando un almacenamiento que crea una "membrana" polarizada. De acuerdo con Sogade et al. (2006), la polarización de membrana es el efecto de PI observado comúnmente en los mapeos de contaminación.

Las modalidades del método de PI generales son: en el dominio del tiempo, en el dominio de la frecuencia, resistividad compleja y dominio espectral. El primero fue practicado por Seigel (1959), el segundo por Hallof (1964), luego con Zonge y Huges (1980), y el último por Pelton et al. (1978).

La cuarta modalidad, el dominio espectral tiene sus orígenes en los experimentos realizados por Vacquier et al. (1957), en los que se concluyó que las mediciones de PI son sensibles a las propiedades capacitivas de las rocas y suelos en bajas frecuencias, que resultan de procesos de difusión en las interfases de los granos minerales y la resistividad del fluido que contiene el poro (Slater y Lesmes, 2002). Esto fue un aporte significativo para la PI debido a que se incluía en la lista de incógnitas del fenómeno, una fuente o agente que lo generaba, que es el fluido en la matriz.

Tradicionalmente la PI se ha aplicado a trabajos relacionados con depósitos minerales (Liu y Peng, 2004); en aplicaciones hidrogeológicas (Ulrich y Slater, 2004), ambientales (Aristodemou y Thomas-Betts, 2000) y estudios que involucran materiales orgánicos (Davidycheva et al., 2006), lo que muestra el interés que hay en la PI a pesar de no contar con una comprensión satisfactoria y total del fenómeno. Este trabajo intenta mostrar las dificultades y potencial del método en problemas de contaminación por hidrocarburos en México.

\section{Fundamentos teóricos}

\subsection{Dominio del tiempo}

A continuación se describirán los fundamentos teóricos del método de polarización inducida empleados para el análisis de los datos del sitio de estudio.

De acuerdo con Seigel (1959), en el dominio del tiempo se define a la cargabilidad con la letra $\mu$

$$
\mu \equiv \frac{E(t)_{t \rightarrow \infty}-E(t)_{t \rightarrow 0}}{E(t)_{t \rightarrow \infty}}
$$

donde $E(t)_{t \rightarrow \infty}$ es el campo eléctrico en estado estacionario y $E(t)_{t \rightarrow 0}$ es el campo eléctrico al cortar la corriente eléctrica.

En la que: $E(t)_{t \rightarrow \infty}-E(t)_{t \rightarrow 0}=e_{x}(t)$ y $E(t)=E_{0}$

Por tanto, la cargabilidad queda expresada como:

$$
\mu=\frac{e_{x}(t)}{E_{0}}
$$

Sin embargo, en la práctica se mide sólo diferencias de potencial y el campo eléctrico es la primera derivada del potencial, por lo cual la expresión (2) se puede reescribir como:

$$
\mu=\frac{\Delta V(t)}{\Delta V(0)}
$$

Orellana (1974) define la cargabilidad en $\mathrm{ms}$ al integrar el decaimiento en el numerador de la ecuación 3 , como se 
muestra en la ecuación 4. El equipo de medición de Iris Instruments (2005), por otro lado, integra tanto el numerador como el denominador de la ecuación 4, llamando a $\mu$ cargabilidad normalizada (ecuación 5) y sus unidades son $\mathrm{mVs} / \mathrm{V}$ o en ms (milesegundos).

$$
\begin{gathered}
\mu=\frac{\int_{0}^{t_{1}} \Delta V(t) d t}{\Delta V(t)_{t=\infty}} \\
\mu_{n}=\frac{1}{\Delta t} \frac{\int_{0}^{t_{1}} \Delta V(t) d t}{\Delta V(t=\infty)}
\end{gathered}
$$

donde $\Delta V(t=\infty)$ es el valor del potencial medido durante el suministro de la corriente y $\Delta t$ es el intervalo de integración.

\subsection{Dominio espectral}

El siguiente modelo (ecuación 6), que se deduce del diagrama de la Figura 1, es el modelo estándar usado por Cole y Cole (Pelton et al., 1978) para describir el comportamiento de polarizaciones dieléctricas y que ha sido de los más usados en la descripción de curvas de decaimiento de potencial.

La impedancia del circuito equivalente de la Figura 1, se expresa como (Pelton et al., 1978):

$$
Z(i \omega)=R_{0}\left[1-m\left(\frac{1}{1+(i \omega \tau)^{c}}\right)\right]
$$

Donde $R_{0}$ es la resistencia asociada a la impedancia de la polarización, $m$ es la cargabilidad y $(i \omega \tau)^{c}$ es el inverso de la reactancia.

Se han desarrollado otras variantes del modelo ColeCole sin embargo, el modelo propuesto por Pelton et al. (1978), es el que mejor describe el fenómeno de polarización observada en la naturaleza, como lo hace notar Nordsiek y Weller (2008).

Si se recurre a la Ley de Ohm y se considera la geometría del arreglo de medición de la impedancia, entonces se puede definir una resistividad como:

$$
\rho(i \omega)=\rho(0)\left[1-m\left[1-\frac{1}{1+(i \omega \tau)^{c}}\right]\right]
$$

Donde $\rho(0)$ es la resistividad, $m$ cargabilidad, $\tau$ tiempo de relajación y $c$ es un exponente.

En la Figura 2 se muestran los modelos para cinco curvas de cargabilidad con valores que van de 0.1 a 0.9 . Las curvas de fase son importantes debido a que, mediante la obtención de la frecuencia crítica $(F c)$, que es la frecuencia a la cual ocurre el máximo cambio de fase, se determina la constante $\tau$, ya que $F c$ es inversamente proporcional a $\tau$ (Van Blaricom, 1992). En cuanto a la amplitud, se observa que ésta disminuye al aumentar la cargabilidad y la frecuencia, esto es concordante con la teoría en el dominio de la frecuencia, en la que se prueba que la resistividad disminuye al aumentar la frecuencia.

Las investigaciones y el trabajo de campo indican que la constante de tiempo $\tau$, es dependiente directamente del tamaño de grano de las partículas y determina la cantidad

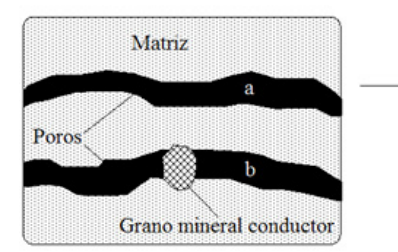

a)

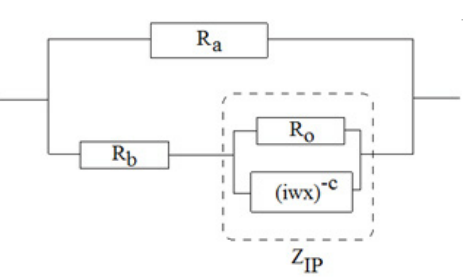

b)
Figura 1. a) Porción de roca mineralizada polarizable y b) circuito eléctrico equivalente (Luo y Zhang, 1998). Donde $(i \omega x)^{c}$ es la reactancia superficial, $R_{a}$ es la resistencia del canal no bloqueado, $R_{b}$ resistencia del conductor iónico y electrónico, $R_{0}$ es la resistencia pura, $Z_{I P}$ es la impedancia del sistema equivalente.
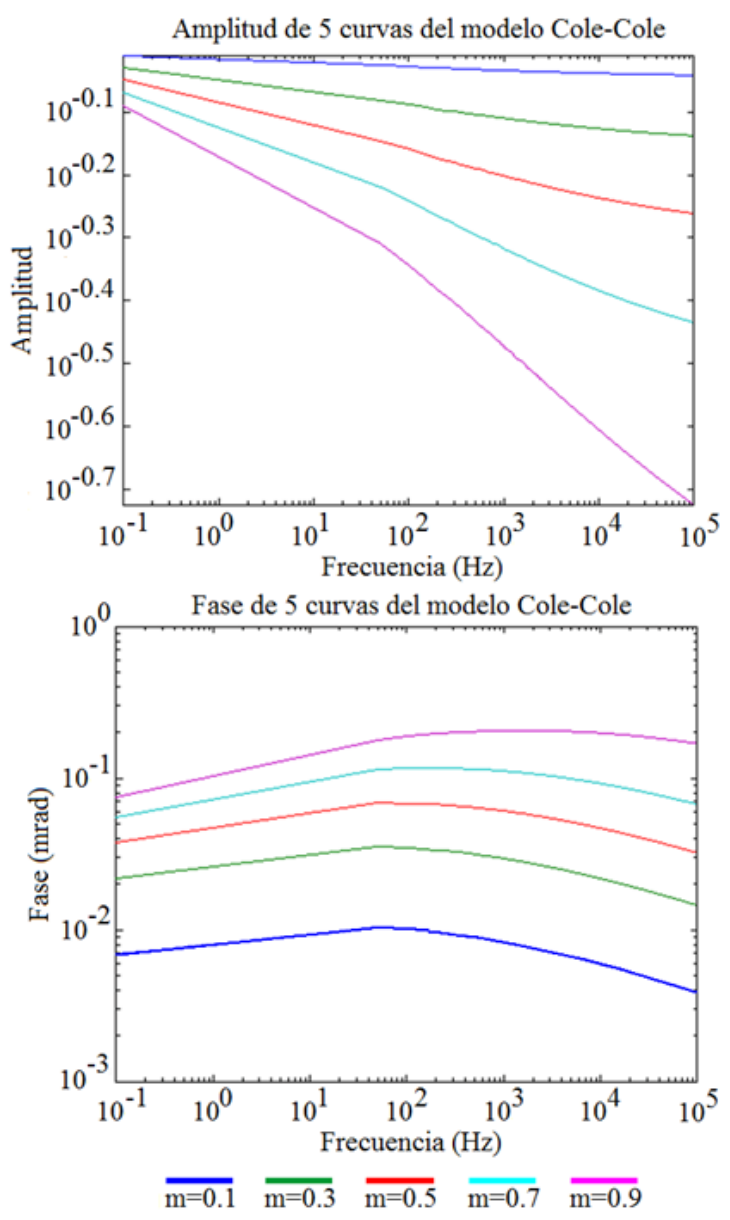

Figura 2. Gráficas de amplitud (arriba) y fase (abajo) de las curvas calculadas del modelo Cole-Cole para cargabilidad con $R_{0}=1, \tau=0.1$ $\mathrm{y} c=0.25$.

de tiempo requerido para el decaimiento del potencial en el dominio del tiempo. Este hecho se ha modelado principalmente para metales (Wong, 1979). En términos generales, se muestra lo referido en la Tabla 1.

El comportamiento de $c$ también ha sido investigado en diferentes medios, obteniéndose rangos desde 0.1 hasta 1. Van Blaricom (1992) dice que el rango es de 0.1 a 0.5 , mientras que el diccionario de la SEG (Sheriff, 2002) 
Tabla 1. Comportamiento cualitativo del parámetro $\tau$ y del parámetro $c$

\begin{tabular}{lc}
\hline Tamaño de grano & $\tau$ \\
\hline $\begin{array}{l}\text { Fino } \\
\text { Grueso }\end{array}$ & $\begin{array}{c}\text { Pequeño } \\
\text { grande }\end{array}$ \\
\hline Tipo de medio & $c$ \\
\hline $\begin{array}{l}\text { Homogéneo } \\
\text { No homogéneo }\end{array}$ & $\begin{array}{c}\text { Grande } \\
\text { Pequeño }\end{array}$ \\
\hline
\end{tabular}

propone hasta 0.6 y Luo y Zhang (1998) encontraron valores de 0.5 a 1 (ver Tabla 1$)$.

\section{Descripción del área de trabajo}

La zona de pruebas está comprendida entre las coordenadas $19.4681^{\circ}$ y $19.4971^{\circ}$ latitud norte y $99.1627^{\circ}$ y $99.2037^{\circ}$ de longitud oeste.

El área de trabajo pertenece a la provincia fisiográfica de la Zona Neovolcánica cuyas rocas del Eje Neovolcánico limitan con o cubren parcialmente la secuencia vulcanosedimentaria del Terreno Guerrero al este, a rocas sedimentarias de la cuenca de Zimapán al norte, las calizas de la provincia de la Sierra Madre Oriental al noroeste y a las calizas de la cuenca Guerrero- Morelos al suroeste (Servicio Geológico Mexicano, 2002).

A nivel superficial, el sitio se encuentra en una zona de relleno de pendiente suave, cubierto por aluvión formado durante el periodo Cuaternario, con depósitos de sedimentos de las secuencias vulcano-sedimentarias del Terreno de Guerrero (al occidente de la carta) y de las rocas sedimentarias de la cuenca de Zimapán (al norte).

\section{Método}

Se realizó una tomografía eléctrica con una línea de 24 electrodos de acero y otra de 24 electrodos impolarizables (Dahlin et al., 2002), todos equiespaciados a $3 \mathrm{~m}$ con arreglo dipolo- dipolo (Figura 3). La programación se realizó para toma de lecturas de resistividad y cargabilidad con una corriente de 200 (mA), 800 (V) en potencial de envío y 200 $(\mathrm{mV})$ en recepción estándar.

El suelo del sitio, en general, se encontraba en condiciones de remediación, mediante el uso de mecanismos como: extracción de fase libre manual y automatizada, inyección de aire y extracción de vapores orgánicos, así como del movimiento de tierra para su tratamiento en calderas. Específicamente, en la línea de tomografía, el contaminante se encontraba en las tres fases (gas, libre y disuelta), siendo visible la recuperación de la fase libre en las zanjas intercomunicadas 4 y 4a (Figura 4), aproximadamente a $7 \mathrm{~m}$ de profundidad.

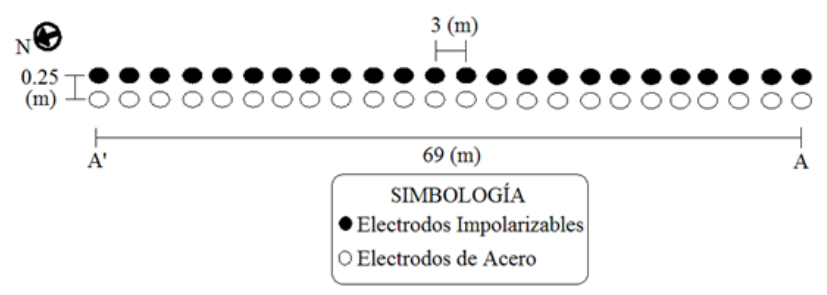

Figura 3. Esquema de la distribución de los electrodos para la toma de lecturas en el sitio contaminado por hidrocarburos.

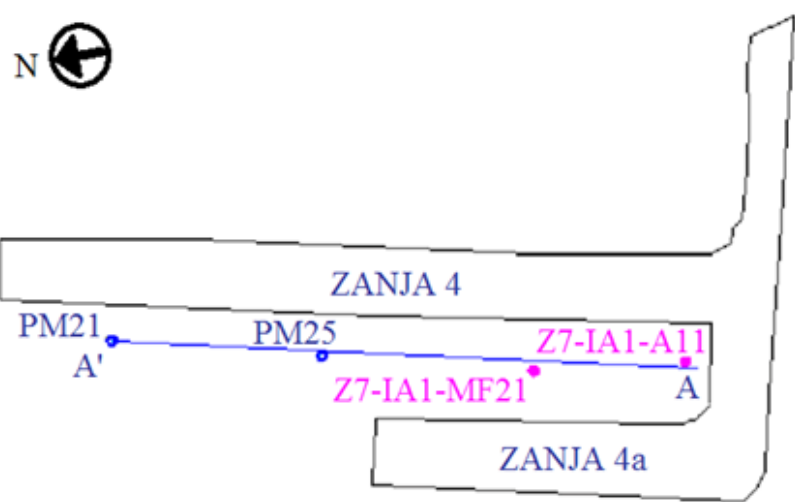

Figura 4. Croquis de la sección A-A' de la tomografía eléctrica (resistividad y PI).

\section{Resultados}

El procesamiento de la información se llevó a cabo con el software de interpretación llamado EarthImager versión 2.4.0, de Resistivity and IP Inversion Software de Advanced Geosciences, Inc. (AGI, 2009).

De los parámetros de polarización inducida $(\tau, \mu$ y el error cuadrático medio - $R M S$, por sus siglas en inglés- del modelo Cole-Cole estándar) sólo el mapa de variación del parámetro $\tau$ se elaboró en Surfer Versión 8.0 mediante la función de interpolación triangular lineal. Estos parámetros son aparentes, ya que se determinaron con el programa Prosys II (Iris Instruments, 2005) directamente de los datos de campo.

La Figura 5 muestra las respuestas de resistividad y cargabilidad invertidas, obtenidas al procesar los 208 datos tomados el día 8 de abril de 2010, mostrando en la parte inferior, la sección A-A' de la línea del tendido con la posición de los pozos cercanos de los cuales se extrajo muestras de agua y de suelo para su análisis en laboratorio, con lo que se obtuvieron las concentraciones de benceno (pozos marcados con un " $\mathrm{X}$ " en las verticales de PM21, PM25, Z7-IA1-MF21 y Z7-IA1-A11).

Estos datos, presentaron un potencial espontáneo (SP por sus siglas en inglés) máximo de $98.3 \mathrm{mV}$ y mínimo de -90.4 mV (Figura 6), una desviación estándar promedio de 21.7, de resistividades aparentes que van desde -4.7 a 


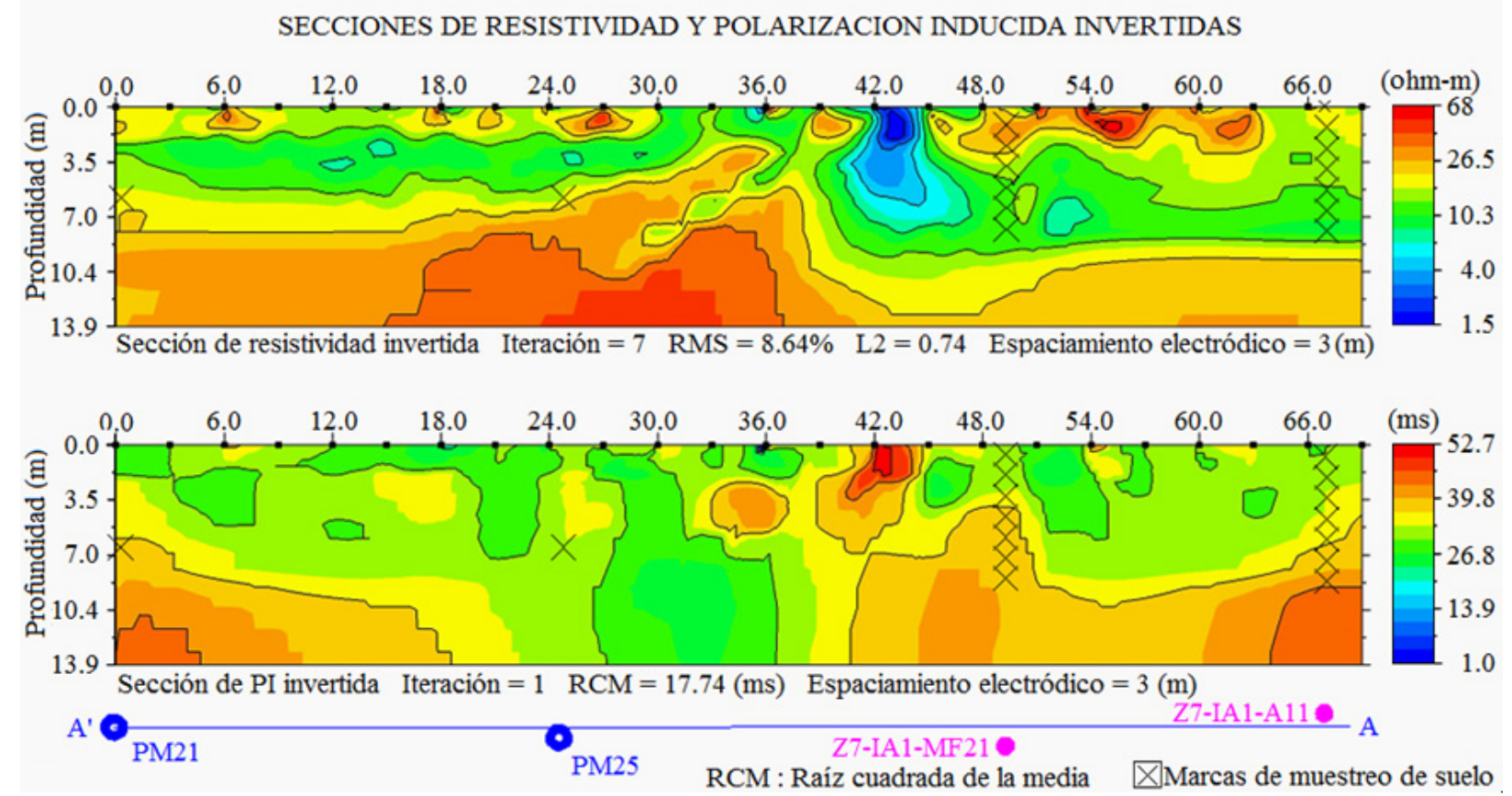

Figura 5. Gráfica del potencial espontáneo obtenido en los datos del sitio contaminado por hidrocarburos.

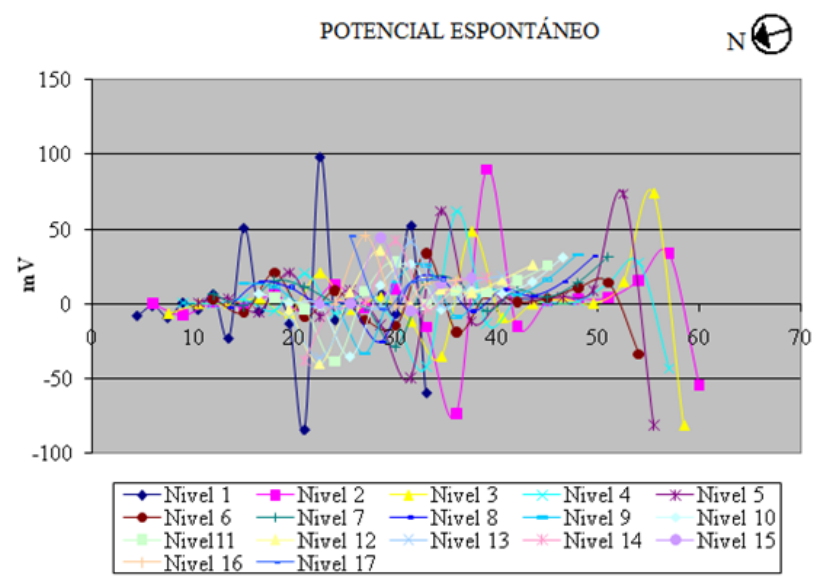

Figura 6. Potencial espontáneo (SP por sus siglas en inglés) en cada uno de los niveles de la sección de tomografía eléctrica.

$42.43 \mathrm{ohm}-\mathrm{m}$. Los datos de resistividad negativa en arreglos colineales no son aceptables y los podemos entender por los cambios del potencial espontáneo altos como el de -34.4 $\mathrm{mV}$ con valores de Vp bajo de $0.93 \mathrm{mV}$. La cargabilidad aparente, por su parte, se encuentra en un rango entre -299.9 y $513.2 \mathrm{~ms}$.

La distribución de la resistividad invertida muestra a una profundidad promedio de $3.5 \mathrm{~m}$, un horizonte de forma tabular con valores bajo-resistivos de alrededor de $10 \mathrm{ohm}-\mathrm{m}$ (en tonos verde y azul), que de acuerdo con la información proporcionada verbalmente, corresponde a un horizonte de almacenamiento de agua somera. Se observa además una zona alto-resistiva en la vertical, bajo las posiciones de 18 a $38 \mathrm{~m}$. En el caso de la imagen de cargabilidad, predominan valores medios (alrededor de 26 $\mathrm{ms}$ en tonos verdes y amarillos) aumentando la cargabilidad en profundidad y en direcciones laterales (esquina inferior izquierda y derecha).

En la zona comprendida entre los pozos Z7-IA1-MF21 y Z7-IA1-A11 para el caso de la cargabilidad de la Figura 5 , en la vertical de los pozos aumenta la cargabilidad al profundizar, con variaciones intermedias en tonalidades verdes y amarillo (con valores entre 20 y $40 \mathrm{~ms}$ ). En la Figura 7, en la misma zona sólo se observa un aumento de la concentración de benceno en el lado inferior izquierdo, por lo que se cuenta con mayor información para realizar muestreo de pozos o instalación de mecanismos de remediación con las imágenes de cargabilidad, ya que se tiene más detalle del área de estudio, a diferencia de la interpretación lineal con datos de muestreos como el que se observa en la Figura 7.

En la respuesta resistiva los pozos PM21 y PM25 tienen valores semejantes (alrededor de los $20 \mathrm{ohm}-\mathrm{m}$ ), a diferencia de los pozos Z7-IAI-IA11 y Z7-IAI-MF21 que responden con valores más conductores (del orden de los $10 \mathrm{ohm}-\mathrm{m}$ ). Por otro lado, en la respuesta de PI, los pozos PM21 y PM25 tienen respuestas distintas y de acuerdo a la Tabla 2, una concentración distinta. Sin embargo, para los pozos Z7-IAI-IA11 y Z7-IAI-MF21, la respuesta de PI son similares (del orden de $37 \mathrm{~ms}$ ) y parecida a la del pozo PM21, pero la concentración de Z7-IAI-IA11 es el doble que la del pozo Z7-IAI-MF21. De los datos obtenidos se puede concluir que el benceno, para la zona, se puede caracterizar con cargabilidad de $37 \mathrm{~ms}$, este hecho necesita 
CONCENTRACIONES DE BENCENO EN SUELO (Pozos Z 7-AIl-All y Z 7-AIl-MF21)
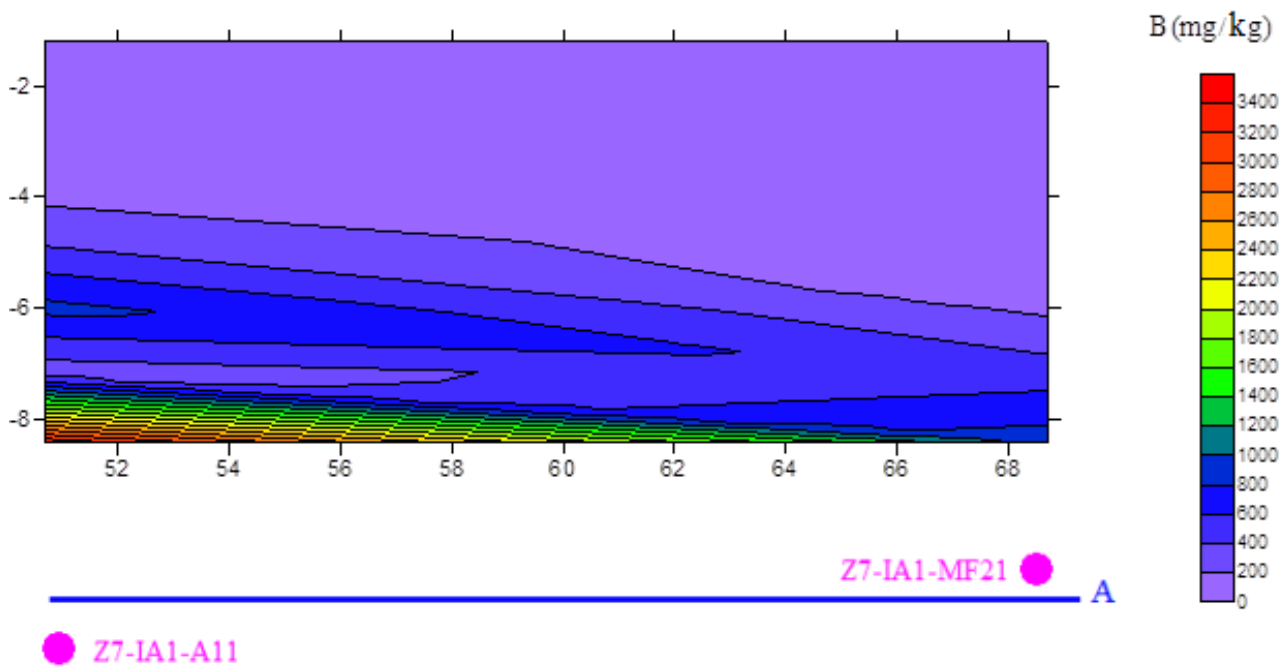

Figura 7. Distribución de concentración de benceno en suelo correspondiente al perfil eléctrico. Profundidades calculadas con los parámetros de Edwards (1977). Interpolación triangular lineal.

Tabla 2. Concentraciones de benceno en agua.

\begin{tabular}{lc}
\hline Pozo & Concentración de benceno $(\mathrm{mg} / \mathrm{L})$ \\
\hline PM-21 & 40.891 \\
PM-25 & 399.753 \\
\hline
\end{tabular}

más investigación para caracterizar un contaminante por su respuesta de PI, en contraste con la resistiva.

En la Tabla 3 de los pozos Z7-IAI-IA11 y Z7-IAI-MF21, se observa que al aumentar la profundidad (de 1.2 a $8.4 \mathrm{~m}$ ) aumenta la concentración del benceno para ambos pozos, sin embargo, los valores obtenidos para Z7-IAI-IA11 son de menores proporciones con respecto a Z7-IAI-MF21, esto es que al aumentar la concentración también aumenta la cargabilidad .

En términos generales y de acuerdo con los datos de laboratorio y las secciones de resistividad y cargabilidad, se encontró que la cargabilidad es directamente proporcional a las concentraciones, como lo establece la teoría (Pelton et al., 1978; Wong, 1979), al menos como se muestra para los pozos Z7- IAI-MF21 y Z7-IAI-IA1, ya que para PM25 y PM21 no se observa esa relación. Esto puede deberse a que pudo haberse cometido algún error en los análisis de laboratorio, ya que el PM25 es el único que no se comporta como los otros tres.

La información adicional de este estudio corresponde a la probable distribución de los diferentes tamaños de grano, que se muestra en la Figura 8, mediante el parámetro $\tau$, que de manera superficial se muestran valores pequeños de $\tau$ (que coinciden con las observaciones de campo) y continúa así en la parte central. En el lado inferior derecho, se observan los mayores valores $\tau$ (la zona en color rojo) y del lado inferior izquierdo, valores intermedios (amarillo
Tabla 3. Concentraciones de benceno en suelo.

\begin{tabular}{ccccccccc}
\hline Muestra & $\begin{array}{c}\text { B 1.20 } \\
(\mathrm{m})\end{array}$ & $\begin{array}{c}\text { B 2.40 } \\
(\mathrm{m})\end{array}$ & $\begin{array}{c}\text { B 3.60 } \\
(\mathrm{m})\end{array}$ & $\begin{array}{c}\text { B 4.80 } \\
(\mathrm{m})\end{array}$ & $\begin{array}{c}\text { B 6.00 } \\
(\mathrm{m})\end{array}$ & $\begin{array}{c}\text { B 7.20 } \\
(\mathrm{m})\end{array}$ & $\begin{array}{c}\text { B 8.40 } \\
(\mathrm{m})\end{array}$ \\
\hline Z7-IAI- IA11 & 0.8 & 0.82 & 19.17 & 21.9 & 157.06 & 507.23 & 896.92 \\
Z7-IAI- MF21 & 0.78 & 3.46 & 57.77 & 362.79 & 860.55 & 265.85 & 3329.74 \\
\hline
\end{tabular}

y verde). Esto parece tener una correlación con la imagen de resistividad (colores azules a verdes en la escala gráfica) ubicados en la parte superficial y hasta los $5 \mathrm{~m}$ de profundidad. De manera que de acuerdo la teoría, en la parte superficial se tiene material de grano más fino y al parecer, en la parte inferior aumenta el tamaño de grano (Tabla 1)

Las secciones del parámetro $\tau$ tienen un gran potencial de desarrollo ya que se tendría una mejor idea de la distribución de materiales finos y gruesos en el medio, que conlleva a entender el comportamiento del contaminante en tiempo y espacio, que es lo que se busca en una caracterización ambiental.

Desafortunadamente, no se pudo adquirir la información litológica de campo para correlacionarlos con los valores del parámetro $c$, sólo se supo de manera verbal que la litología era sumamente complicada debido a que en cada pozo los horizontes no guardaban correlación.

\section{Conclusiones}

- Las respuestas de PI en el dominio del tiempo aportan información, que junto con la de resistividad, permiten una mejor comprensión e interpretación de problemas en contaminación.

- La PI es una herramienta que ayuda a mejorar los modelos de predicción de movilidad de contaminación 


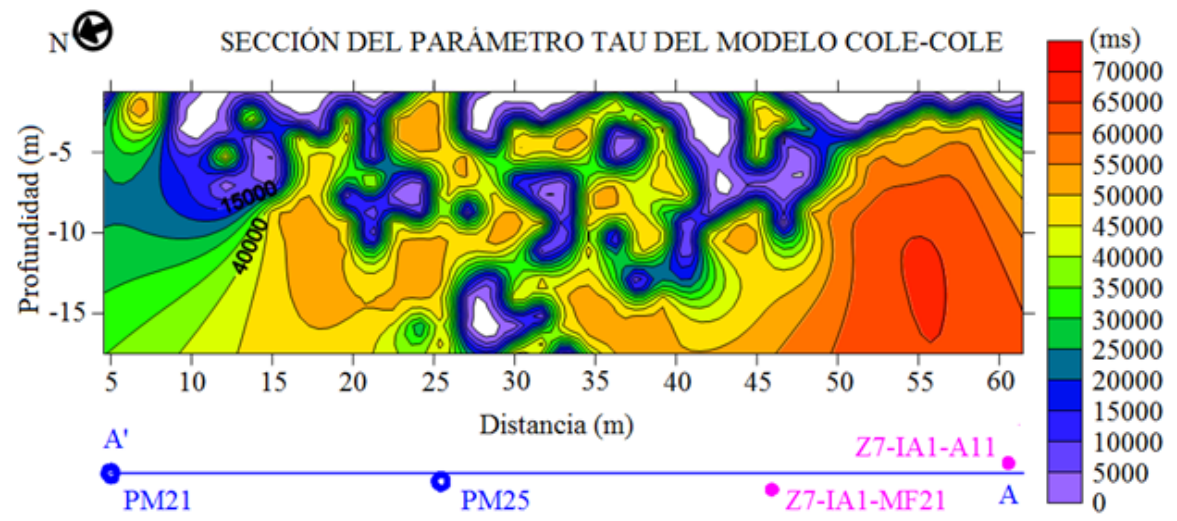

Figura 8. Sección de la distribución del parámetro $\tau$ aparentes del modelo Cole-Cole estándar, obtenida con el método de Kriging. Profundidades calculadas con los parámetros de Edwards (1977).

con una estratigrafía complicada.

- De los modelos Cole-Cole y sus variantes, se puede obtener información de tipo granulométrico $(\operatorname{con} \tau)$ y la homogeneidad del medio $(\operatorname{con} c)$, de manera que se puedan correlacionar con la respuesta de cargabilidad y resistividad a priori, que pueden ser de gran utilidad en el muestreo direccionado para la caracterización de sitios contaminados.

- Los valores de SP negativos pueden ser los generadores de curvas muy ruidosas; sin embargo, el empleo del dispositivo electródico, la secuencia en las lecturas, la elección de los electrodos y los cuidados en campo siguen siendo de suma importancia, ya que como se mencionó anteriormente, los datos de PI son muy susceptibles al ruido ambiental, el cual debe reducirse lo más posible.

\section{Agradecimientos}

Los autores agradecen a Víctor Manuel Sánchez Granados por haber proporcionado las facilidades para realizar las pruebas en el sitio contaminado, a David Escobedo Zenil, Jefe del Departamento de Geofísica de la Facultad de Ingeniería por otorgar los permisos pertinentes, a Cecilia Delgado Solórsano y Diana Yael Pérez por haber colaborado durante los trabajos de adquisición, así como a Juan Carlos Uresti por sus acertadas observaciones.

\section{Referencias}

Advanced Geosciences, Incorporated (AGI), 2009, Instruction Manual for Earth Imager 2D Version 2.4.0 Resistivity and IP Inversion Software, $139 \mathrm{p}$.

Aristodemou, E., Thomas-Betts, A., 2000, DC resistivity and induced polarisation investigations at a waste disposal site and its environments: Journal of Applied Geophysics, 44, :275-302.

Dahlin, T., Leroux, V., Nissen, J., 2002, Measuring techniques in induced polarization Imaging: Journal of Applied Geophysics, 50, 279-298.

Davydycheva, S., Rykhlinsky, N., Legeido, P., 2006, Electrical-Prospecting method for hydrocarbon search using the induced-polarization effect: Geophysics, 71, 4, G179-G189.

Edwards, L.S., 1977, A modified pseudosection for resistivity and PI: Geophysics, 42,1020-1036.

Hallof, P., 1964, A comparison of the various parameters employed in the variable-frequency induced-polarization method: Geophysics, $3,425-433$.

Iris Instruments, 2005, User's Manual of Syscal Pro Standard \& Switch, $48-72$ or 96 Version, 10 channels Resistivity-meter for Resistivity and IP measurements, $85 \mathrm{p}$.

Liu, L., Peng, S., 2004. , Prediction of hidden ore bodies by synthesis of geological, geophysical and geochemical information based on a dynamic model in Fenghuangshan ore field, Tongling district, China: Journal of Geochemical Exploration, 81: , 81-98.

Luo, Y., Zhang, G., 1998, Theory and Application of Spectral Induced Polarization: Tulsa, Oklahoma, Society of Exploration Geophysicists, $171 \mathrm{p}$.

Nordsiek, S., Weller, A., 2008, A new approach to fitting inducedpolarization spectra: Geophysics, 73, F235-F245.

Orellana, E., 1974, Prospección Geoeléctrica por campos variables: Madrid, Paraninfo, $571 \mathrm{p}$.

Pelton, W. H., Ward, S. H., Hallof, P. G., Sill, W. R., Nelson, P. H., 1978, Mineral Discrimination and removal of inductive coupling with multifrequency IP: Geophysics, 43, 588-609.

Seigel, H.O., 1959, Mathematical Formulation and type curves for Induced Polarizacion: Geophysics, 24, 547-565.

Servicio Geológico Mexicano (SGM), 2002, Carta Geológico-Minera Ciudad de México E14-2, Estado de México, Tlaxcala, Distrito Federal, Puebla, Hidalgo y Morelos., escala 1:250000: Pachuca, Hidalgo., Primera Edición.

Sheriff, R.E., 2002. Encyclopedic Dictionary of Applied Geophysics: Society of Exploration Geophysicist, 13 Geophysical References Series , Fourth Edition, 429 p., CD-ROM.

Slater, L., Lesmes, D., 2002, IP interpretation in enviromental investigations: Geophysics, 67, 77-88.

Sogade, J.A., Scira-Scappuzzo, F., Vichabian, Y., Shi, W., Rodi, W., Lesmes, D.P., Morgan, F.D., 2006, Induced-polarization detection and mapping of contaminant plumes: Geophysics, 71, B75-B84.

Ulrich, C., Slater, D., 2004, Induced polarization measurements on unsaturated, unconsolidated sands: Geophysics, 69, 762-771.

Vacquier, V, Holmes, C.R., Kintzinger, P.R., Lavergne, M., 1957. Prospecting for ground water by induced electrical polarization: Geophysics, 22: 660-687.

Van Blaricom, R., 1992, Practical Geophysics II for the Exploration Geologist: U.S.A., Northwest Mining Association, 570 p.

Wong, J., 1979, An electrochemical model of the induced-polarization phenomenon in disseminated sulfide ores: Geophysics, 44, 12451265. 
Zonge, K., Hughes, L., 1980, The complex resistivity method (en línea), $\quad$ Manuscrito recibido: Junio 3, 2011.

Tucson AZ, USA, <http://www.zonge.com/FieldCR.html>, Manuscrito corregido recibido: Abril 20, 2012.

consultado 30 de septiembre de 2010.

Manuscrito aceptado: Mayo 24, 2012. 